\title{
An Iterated Local Search for the Split Delivery Vehicle Routing Problem
}

\author{
Z.Z. Wen, X.Y. Dong, S. Han \\ Beijing Key Lab of Traffic Data Analysis and Mining, School of Computer and IT \\ Beijing Jiaotong University \\ Beijing, China
}

\begin{abstract}
A multi-restart iterated local search (MRSILS) algorithm is introduced for the Split Delivery Vehicle Routing Problem (SDVRP). The initial solution is generated by the GENIUS and applied the local search procedure by removing one node from its current route and inserting it into the best locations, with the possibility of splitting its demand. A node inserting algorithm is proposed for SDVRP, trying to find a better solution by an insertion with the consideration of splitting the demand. The perturbation is applied while certain predefined continuous no improvement local search steps are occurred. In order to extend the search space and keeping the quality of the restart solution, a method is designed for the perturbation that the perturbed solution is chosen from an elite solution set, while the best solution is preferred. The object of this work is to minimize the total travel distance. Experimental results on a benchmark set show that the MRSILS is competitive with a state of the art algorithm.
\end{abstract}

\section{Keywords-SDVRP; local search; perturbation; multi-restart}

\section{INTRODUCTION}

Vehicle Routing Problem with split delivery (SDVRP) is a variant of capacitated vehicle routing problem (CVRP). The demand of each node in SDVRP can be served by more than one vehicle. In SDVRP, there is a set of nodes $N$ with service demand, infinite homogeneous vehicles $V$ with capacity constraint $k$ to provide service and one depot $D$. The vehicles start and end their tours at the depot withoutthe load surpassingkduring the tour and the demand of all the nodes must be satisfied[1].

Compared with other VRPs, SDVRP problem has not been enough concerned. There are still some excellent works published. Dror and Trudeau [1], [2]analyze the savings achieved by allowing deliveries split in some VRP instances and provide a heuristic algorithm for SDVRP. Nowak et al. [3] show the potential benefit of split loads and prove the most benefit occurring with delivery of node just one half of vehicle capacity. Drorand Trudeau [1] describe valid inequalities for the SDVRP and Archetti et al. [4]describe a tabu search algorithm for SDVRP. Chen et al. [5] and Archetti et al. [6] put forward hybrid heuristics for SDVRP combined with the advantages ofheuristic and optimization methods to improve calculation efficiency of the single algorithm. Derigset al.[7] present several local-search-based metaheuristics including simulated annealing (SA), threshold accepting (TA), record-torecord travel (RRT), the attribute based hill climber heuristic $(\mathrm{ABHC})$ and the attribute based local beam search heuristic (ABLBS) for the SDVRP, and the ABHC get the best experimental results.
Boudia et al. [8] point out that the using of dynamic programming increases the running time by $30 \%$ without significant improvement than the one found by a greedy heuristic.In this work, we propose a greedy iterated local search (ILS) algorithm in which the insertion move in local search phase follows the simple principle that always do the best insertion, considering the possible splitting of the demand. In order to extend the search space effectively, without deteriorating the quality of the restart solution, a multi-restart perturbation method is designed, which is derived from the idea of Dong et al. [9].

The rest of this paper is organized as follows. In section 2, we present our multi-restart local search algorithm, including the method for the initial solution, local search structure and perturbation method. In section 3 , the experimental resultsare reported and analyzed. In section 4, we put forward the innovation and deficiency point of our algorithm.

\section{MULTI-RESTART ITERATED LOCAL SEARCH ALGORITHM}

The followingtermsare used in this paper. The number of nodes is $N$. Direct trip is a tour in which a vehicle starts from the depot, goes directly to a customer, delivers $k$ units and then turns back directly to the depot [4]. Remove savingof nodeiis denoted by $R S_{i}$ and computed by $R S_{i}=\sum_{r \in R(i)}\left(c_{p i}+c_{i q}-c_{p q}\right)$, where $R(i)$ is all the cars that visit node $i$ in current solution; $p$ and $q$ are the predecessor and the successor of node $i$ in route $r$; $c_{p i}$ denotes the distance between node $p$ and $i$ and so on. Remove saving list $R S L$ is defined as a non-increasing list of $R S_{i}$ for all node $i$. The minimum cost for inserting node $i$ into route $r$ is denoted by $I C_{i r}=\min \left\{c_{s i}+c_{i t}-c_{s t}\right\}$, where $s$ and $t$ are two adjacent nodes in route $r$. The minimum costs for inserting node $i$ into all the routes in $R$ formingthe insert cost list of node $i$, denoted by $I C L_{i}$, and the elements in which are sorted in ascending way.

There are two phases in local search. In phase 1, if the demands of some nodes are larger than $k$, then the nodes will be divided into two parts $I_{K}$ and $I_{R}$. Part $I_{K}$ contains the nodes with demand surpassing $k$, and the demand of the node $i$ in $I_{K}$ is set to $k\left\lfloor d_{i} / k\right\rfloor$. Then nodes are created as $I_{K}{ }^{\prime}$ for the nodes in $I_{K}$ with non-zero remaining demand. Part $I_{R}$ is the remaining nodes by removing $I_{K}$ from $I$, i.e. $I_{R}=N_{K}$. Initial solution for $I_{K}$ is generated by adding direct trips for every node. We use GENIUS [10] for $I_{R} \cup I_{K}{ }^{\prime}$ to generate a big TSP tour, and then split the tour by $k$. The initial solution consists of the above two initial solutions. 
In phase 2, we propose a multi-restart iterated local search for SDVRP. We do the local search by removing node iand then reinserting it into another position according to the method presented in section 2.1. The searching order of node $i$ is arranged by $R S L$. This arrangement is based on the hypothesis that searching the node with larger remove saving can achieve a better solution with greater possibility. A qualified solution pool is also maintained to enlarge the restart-solution space. The pool is designed for the perturbation function, which is the solution chosen from the pool is perturbed to generate the start solution for the next local search. The algorithm is names MRSILS and the pseudo-code is shown in algorithm 1, where $s_{0}$ is the initial solution; $s^{\prime}$ is the current solution; $s^{*}$ is the current best solution; $s^{\prime \prime}$ is the solution which is the result of local search starting from $s^{\prime}$; the number of nodes is denoted by $N$; Sis the solution poolstoring a set of solutions; pool_sizerepresents the maximum size of $S$.

Algorithm 1: Multi-restart local search frame

1. Generate $s_{0}, s^{\prime} \leftarrow s_{0}, s^{*} \leftarrow s_{0}$, $i$, flag $\leftarrow$ false, $S \leftarrow \phi$, pool_size;

2. while (termination criterion is not satisfied) do

3. Compute the $R S L$ for $s^{\prime}$;

4. $i \leftarrow 0$;

5. while $(i<N)$ do

6. $s^{\prime \prime}=\operatorname{LocalSearch}\left(s^{\prime}, i\right)$;

7. if $s^{\prime \prime}$ is better than $s^{\prime}$ then

8. $s^{\prime} \leftarrow s^{\prime \prime}$;

9. endif

10. if $s^{\prime}$ is better than ${ }^{s^{*}}$ then

11. $s^{*} \leftarrow s^{\prime}$, flag $\leftarrow$ true;

12. endif

13. if the perturbation limitation is met then

14. ifflag is true then

15. $S \leftarrow \phi$, flag $\leftarrow$ false;

16. endif

17. $S \leftarrow \operatorname{accept}\left(S, s^{\prime}\right)$;

18. $s^{\prime} \leftarrow \operatorname{perturb}\left(S, s^{*}\right)$;

19. endif

20. $i++$;

21. endwhile

22. endwhile

23. Stop and output $s^{*}$.

In step 17 of the algorithm 1 , the $\operatorname{accept}\left(S, s^{\prime}\right)$ is used to estimate whether s' is qualified to be added to $S$ : if the size of $S$ is less than pool_size, add s'to S; otherwise if s' is better than the worst one in $\mathrm{S}$, then remove the worst and insert s' into S. The solutions in Sare several good solutions around the current best solution $\mathrm{s}^{*}$, so choosing a solution from it to generate the restart solution is a reasonable option. The perturbation method in step 18 is shown in section 2.2.

\section{A. Insertion Method}

In the localsearch, we successively remove a node from the current solution and reinsert it to its best location. The insertion is designed as follows to support the possible split operations. As for the selection of the least cost move of node $i$, all the routes with surplus capacity larger than the demand of node $i$ are checked, in addition to the combination of several routes with least insertion cost. The whole insertion procedure is shown as below.

Step1: Remove node $i$ from s' and compose route list $R L_{i}$;

Step 2: Compute the insertion cost $I C_{i r}$ for route $r$ in $R L_{i}$, whose surplus capacity can satisfy the demand of node $i$, denote the route with minimum $I C_{i r}$ by $r_{\min }$, and set $\min _{i c}$ to $I C_{i r}$; if it does not exist, set $m i n_{i c}$ to a very large number;

Step 3: Compute the minimum cost $\min _{i s}$ for the case of the sum of the surplus capacity of the first $z$ routes in $R L_{i}$ is larger than the demand of node $i$, while the first $z-1$ routes can not satisfy the demand, where the routes considered above have most least insertion cost and the surplus capacity of each one is smaller than the demand of node $i$; if such case does not exist, the $\min _{i s}$ is a very large number;

Step 4: Insert the node $i$ to the case indicated by the smaller $\min _{i s}$ and $\min _{i c}$;

\section{B. Perturbation Strategy}

The perturbed solution and the perturbation method have a great influence on the quality of the final best solution. There are two steps for the perturbation strategy. Firstly choose the solution which will be perturbed; secondly perturb the chosen solution. As for the first step, when the $S$ is full, we choose one randomly from it; otherwise the current best solution $s^{*}$ is chosen. The perturbation method is shown as algorithm 2 .

Usually, perturbation is occurred when the local search is trapped in a local optimum, i.e. the current solution can not be improved by re-inserting any node.

\section{Algorithm 2: Perturbation strategy}

1. Choosing two routes $r_{1}$ and $r_{2}$ from the solution being perturbed randomly;

2. Generate start nodes $A_{i}$ and $B_{i}$ for these two routes randomly;

3. Randomly generate $C_{A}$ the numberof being perturbed nodes for $r_{1}$;

4. Mark $C_{A}$ nodes starting from $A_{i}$ on path $r_{1}$ as set $N_{R A}$;

5. Suppose the total demand of node set $N_{R}$ is denoted by $d_{R}$, and the surplus capacity of route $r$ is denoted by $s_{r}$, then take the maximal number of nodes in $r_{2}$ starting from $B_{i}$ as $N_{R B}$, satisfying $s_{r 1}+d_{R A}-d_{R B}>0$;

6. If all of the nodes in $N_{R A}$ can be served by $r_{2}$, the perturbation can be implemented by exchanging the $N_{R A}$ and $N_{R B}$ directly ; otherwise, exchange the $N_{R A}$ and $N_{R B}$ with the splitting of the last node in $N_{R A}$.

However, it can be assumed that the local search can hardly improve the solution further after checking certain number of nodes without improvement, and so make a perturbation to continue the local search is reasonable in such a case. We set the above number of insertion nodes as the criterion for the perturbation in step 13 in algorithm 1 , which is denoted by $L$.

\section{EXPERIMENTAL RESULTS}

The problems 1-5 and 11 from Gendreauet al.[11] are considered in this work. The customer number of these instances is between 50 and 199. As proposed by Archetti et al.[4], the demand of the customers is changed by introducing 
two parameters $\alpha$ and $\gamma$, where $\alpha$ and $\gamma$ in the interval $[0,1]$ and $\alpha \leq \gamma$. The demand $d_{i}$ of customer $i$ is set to $d_{i}=\alpha k+\delta(\gamma-\alpha) k$, where $\delta$ is a randomly number in $[0,1]$. The $(\alpha, \gamma)$ are set as follows: $(0.1,0.3),(0.1,0.5),(0.1,0.9),(0.3,0.7),(0.7,0.9)$. The instance set used in this work is provided warmheartedly by $\mathrm{C}$. Archetti, and it is the same with what is used in Derigset al. [7]. We get the results with $1.3 \mathrm{GHz}$ CPU with $3 \mathrm{~GB}$ memory and the algorithm is implemented in $\mathrm{C}++$.

In order to optimize the perturbation, the parameter $L$ is set to $\lceil 0.01 N\rceil,\lceil 0.1 N\rceil,\lceil 0.5 N\rceil$ and $N$, respectively. The MRSILS is run 5 independent times for each case with the five CPU minutes. The best solution is chosen from these five runs, and the comparison is shown in fig. 1 in average relative percentage deviation (ARPD) over the case of $0.01 \mathrm{~N}$, and the RPD is computed as:

$$
R P D=\left(f-f_{0.01 N}\right) / f_{0.01 N} \times 100
$$

where $f$ denotes the objective achieved by the compared case, and $f_{0.01 N}$ denotes the objective gotten by setting the $L$ to $0.01 N$. From this figure, it can be seen that the results are generally the best with $L=0.01 \mathrm{~N}$, other than the setting of $L=0.1 \mathrm{~N}$ on the original benchmark set, in which case the setting of $L=$ $0.1 N$ is the best. In the following experiment, the $L$ is set to $0.01 N$.

The ABHC proposed by Derigset al. [7] is one of the state of the art algorithms. In their work, the algorithm is run five times independently with 5, 10, 15, 30 and $60 \mathrm{CPU}$ minutes, respectively, and they report the best results among these runs. They carried out the experiments on $3 \mathrm{GHz}$ PCs with 2 GB memory. In order to make a relative fair comparison, the MRSILS is run in thesame settings and the comparison results are listed in Table 1, where NV denotes the number of vehicles, TD denotes the total distance, and TD\% denotes the relative percentage deviation of the MRSILS over the ABHC. The results show that the MRSILS is comparable to the $\mathrm{ABHC}$, with better for 17 out of 36 instances, and better in overall performance.

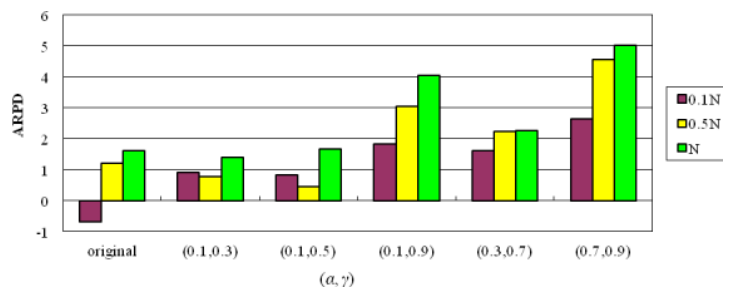

FIGURE I. OPTIMIZATION OF $L$

TABLE I. COMPARISON RESULTS FOR INSTANCES FROM ARCHETTIET AL.[6]

\begin{tabular}{|c|c|c|c|c|c|c|c|c|}
\hline \multicolumn{4}{|c|}{ Instance } & \multicolumn{2}{|r|}{$\mathrm{ABHC}$} & \multicolumn{3}{|c|}{ ILS } \\
\hline Name & $|C|$ & $\alpha$ & $\gamma$ & $\mathrm{NV}$ & $\mathrm{TD}$ & $\mathrm{NV}$ & TD & TD\% \\
\hline $\mathrm{P} 01$ & 50 & - & - & 5 & 524.61 & 5 & 531.03 & -1.22 \\
\hline P02 & 75 & - & - & 10 & 829.89 & 10 & 831.85 & -0.24 \\
\hline P03 & 100 & - & - & 8 & 826.14 & 8 & 834.52 & -1.02 \\
\hline P04 & 150 & - & - & 12 & 1028.42 & 12 & 1066.04 & -3.66 \\
\hline P05 & 199 & - & - & 16 & 1302.90 & 17 & 1343.67 & -3.13 \\
\hline P11 & 120 & - & - & 7 & 1042.12 & 7 & 1048.00 & -0.57 \\
\hline P01 & 50 & 0.1 & 0.3 & 11 & 776.42 & 11 & 764.90 & 1.48 \\
\hline P02 & 75 & 0.1 & 0.3 & 16 & 1123.98 & 16 & 1130.05 & -0.54 \\
\hline P03 & 100 & 0.1 & 0.3 & 22 & 1478.59 & 22 & 1487.30 & -0.59 \\
\hline P04 & 150 & 0.1 & 0.3 & 32 & 2055.18 & 32 & 2060.06 & -0.24 \\
\hline P05 & 199 & 0.1 & 0.3 & 40 & 2540.06 & 41 & 2546.80 & -0.27 \\
\hline P11 & 120 & 0.1 & 0.3 & 27 & 2913.09 & 27 & 2948.99 & -1.23 \\
\hline P01 & 50 & 0.1 & 0.5 & 16 & 1012.56 & 16 & 1019.95 & -0.73 \\
\hline P02 & 75 & 0.1 & 0.5 & 24 & 1508.73 & 24 & 1512.78 & -0.27 \\
\hline P03 & 100 & 0.1 & 0.5 & 33 & 2035.91 & 33 & 2026.81 & 0.45 \\
\hline P04 & 150 & 0.1 & 0.5 & 49 & 2912.08 & 49 & 2895.16 & 0.58 \\
\hline P05 & 199 & 0.1 & 0.5 & 63 & 3581.66 & 63 & 3579.67 & 0.06 \\
\hline P11 & 120 & 0.1 & 0.5 & 41 & 4270.38 & 41 & 4299.16 & -0.67 \\
\hline P01 & 50 & 0.1 & 0.9 & 26 & 1489.64 & 27 & 1495.85 & -0.42 \\
\hline P02 & 75 & 0.1 & 0.9 & 41 & 2340.09 & 41 & 2317.31 & 0.97 \\
\hline P03 & 100 & 0.1 & 0.9 & 56 & 3145.33 & 56 & 3133.99 & 0.36 \\
\hline P04 & 150 & 0.1 & 0.9 & 84 & 4638.74 & 84 & 4618.62 & 0.43 \\
\hline P05 & 199 & 0.1 & 0.9 & 105 & 5669.26 & 105 & 5635.13 & 0.60 \\
\hline P11 & 120 & 0.1 & 0.9 & 67 & 6890.39 & 67 & 6884.85 & 0.08 \\
\hline P01 & 50 & 0.3 & 0.7 & 26 & 1488.28 & 26 & 1497.15 & -0.60 \\
\hline P02 & 75 & 0.3 & 0.7 & 39 & 2243.93 & 39 & 2253.36 & -0.42 \\
\hline P03 & 100 & 0.3 & 0.7 & 53 & 3014.08 & 53 & 3020.04 & -0.20 \\
\hline P04 & 150 & 0.3 & 0.7 & 79 & 4435.95 & 79 & 4376.86 & 1.33 \\
\hline P05 & 199 & 0.3 & 0.7 & 102 & 5541.09 & 102 & 5516.43 & 0.45 \\
\hline
\end{tabular}




\begin{tabular}{lllllllll} 
P11 & 120 & 0.3 & 0.7 & 64 & 6671.04 & 64 & 6704.80 & -0.51 \\
P01 & 50 & 0.7 & 0.9 & 41 & 2174.54 & 41 & 2164.65 & 0.46 \\
P02 & 75 & 0.7 & 0.9 & 61 & 3266.78 & 61 & 3240.56 & 0.80 \\
P03 & 100 & 0.7 & 0.9 & 82 & 4447.47 & 82 & 4406.68 & 0.92 \\
P04 & 150 & 0.7 & 0.9 & 122 & 6467.17 & 122 & 6447.69 & 0.30 \\
P05 & 199 & 0.7 & 0.9 & 161 & 8297.71 & 162 & 8283.38 & 0.17 \\
P11 & 120 & 0.7 & 0.9 & 98 & 10233.37 & 99 & 10239.82 & -0.06 \\
total & & & & & 114217.55 & & 114163.9 & 0.05 \\
\hline
\end{tabular}

\section{CONCLUSION}

When using local search methods, an intensive exploration of the solution space is performed by moving at each step from the current solution to another promising solution in its neighbors[12]. The proposed MRSILS removes a node and reinserts it to the best location according to SDVRP constraints. It performs well, achieves good solution on standard SDVRP data set[6], and gets the best solution for 17 instances. It is also easy to implement.

\section{ACKNOWLEDGMENTS}

Prof. Y. Lin provides lots of support. Thanks to Prof. C. Archetti for the data set and the help of U. Derigs. This work is supported by The FundamentalResearchFunds for the Central Universities of China (Project Ref. 2014JBM034, Beijing Jiaotong University).

\section{REFERENCES}

[1] Dror M. and P. Trudeau, Savings by split delivery routing. TransportationScience, 23, pp. 141-145, 1989.

[2] Dror M. and P. Trudeau, Split delivery routing. NavalRes.Logistics, 37, pp. 383-402, 1990.

[3] Nowak M, Ergun O, White C, Pickup and Delivery with Split Loads. TransportationScience, 42, pp. 32-43, 2008.

[4] Archetti C, Speranza M and Hertz A, A tabu search algorithm for the split delivery vehicle routing problem. TransportScience, 40, pp. 64-73, 2006.

[5] Chen S, Golden B and Wasil E, The split delivery vehicle routing problem: Applications, algorithms, test problems, and computational results. JournalofNetworks, 49, pp. 318-329, 2007.

[6] Archetti C, Speranza M and Savelsbergh M, An optimization based heuristic for the split delivery vehicle routing problem. TransportScience, 42, pp. 22-31, 2008.

[7] U Derigs, B Li and U Vogel, Local search-based metaheuristics for the split delivery vehicle routing problem.Journalofthe OperationalResearchSociety, 61, pp. 1356-1364, 2010.

[8] Boudia M., Prins C., Reghioui M., An effective memetic algorithm with population management for the split delivery vehicle routing problem. LectureNotesinComputerScience, 4771, pp. 16-30, 2007.

[9] Xingye Dong, Houkuan Huang and Maciek Nowak, A Multi-Restart Iterated Local Search Algorithm for the Permutation Flow Shop Problem Minimizing Total Flow Time.ComputerOperationalResearch, 40(2), pp. 627-632, 2013.

[10] Gendreau, M., A. Hertz, G. Laporte, New insertion and postoptimization procedures for the traveling salesman problem. OperationalResearch, 40, pp. 1086-1094, 1992.

[11] Gendreau M., A. Hertz, G. Laporte, A tabu search heuristic for the vehicle routing problem. ManagementScience, 40, pp. 1276-1290, 1994.

[12] Xingye Dong, Houkuan Huang and Ping Chen, An iterated local search algorithm for the permutation flowshop problem with total flowtime criterion.ComputerOperationalResearch, 36(5), pp. 16641669, 2008. 